\title{
Quantum criticality of the two-channel pseudogap Anderson model: Universal scaling in linear and non-linear conductance
}

\author{
Tsan-Pei $\mathrm{Wu}^{1}$ and Chung-Hou Chung ${ }^{1,2}$ \\ ${ }^{1}$ Electrophysics Department, National Chiao-Tung University, \\ HsinChu, Taiwan, 300, R.O.C. \\ ${ }^{2}$ National Center for Theoretical Sciences, \\ HsinChu, Taiwan, 300, R.O.C.
}

(Dated: November 5, 2018)

\begin{abstract}
The quantum criticality of the two-lead two-channel pseudogap Anderson model is studied. Based on the non-crossing approximation, we calculate both the linear and nonlinear conductance of the model at finite temperatures with a voltage bias and a power-law vanishing conduction electron density of states, $\rho_{c}(\omega) \propto\left|\omega-\mu_{F}\right|^{r}(0<r<1)$ near the Fermi energy $\mu_{F}$. Equilibrium and nonequilibrium quantum critical properties at the two-channel Kondo (2CK) to local moment (LM) phase transition are addressed by extracting universal scaling functions in both linear and non-linear conductances, respectively. Clear distinctions are found on the critical exponents between linear and non-linear conductance. The implications of these two distinct quantum critical properties for the non-equilibrium quantum criticality in general are discussed.
\end{abstract}

PACS numbers:

\section{INTRODUCTION.}

Quantum phase transitions (QPTs) [1], the zerotemperature phase transitions due to quantum fluctuations, are of fundamental importance in condensed matter systems. Near the transitions, these systems show non-Fermi liquid behaviors manifested in universal power-law scaling in all thermodynamic observables. Recently, QPTs in quantum impurity problems [2], have attracted much attention recently due to their relevance for the nano-systems, such as: quantum dots 3], realized experimentally. The well-known Kondo effect [4], the antiferromagnetic spin correlations between impurity and conduction electrons, plays a crucial role in understanding their low temperature behaviors. New scaling laws are expected to occur when the QPTs are associated with the Kondo breakdown in these systems either in equilibrium (at finite temperatures) or under non-equilibrium conditions (at finite voltage bias). Of particular interest lies in QPTs out of equilibrium [5] where distinct universal scalings are expected in contrast to the counterparts in equilibrium.

A fascinating playground to address this issue is the exotic two-channel Kondo (2CK) [6 11] systems with nonFermi liquid ground state due to overscreening of $s=1 / 2$ impurity spin by two independent conduction reservoirs. Much of the theoretical effort has been made for the 2CK physics, including: via Bethe ansatz 12], conformal field theory [13], bosonization 14] and the numerical renormalization group [15]. Experimentally, the 2CK ground state has been realized in semiconductor quantum dots [16], magnetically doped nanowires, and metallic glasses [17, 18]. Recently, Kondo physics in magnetically doped graphene has attracted much attention for the possible $2 \mathrm{CK}$ physics as well as the pseudogap local

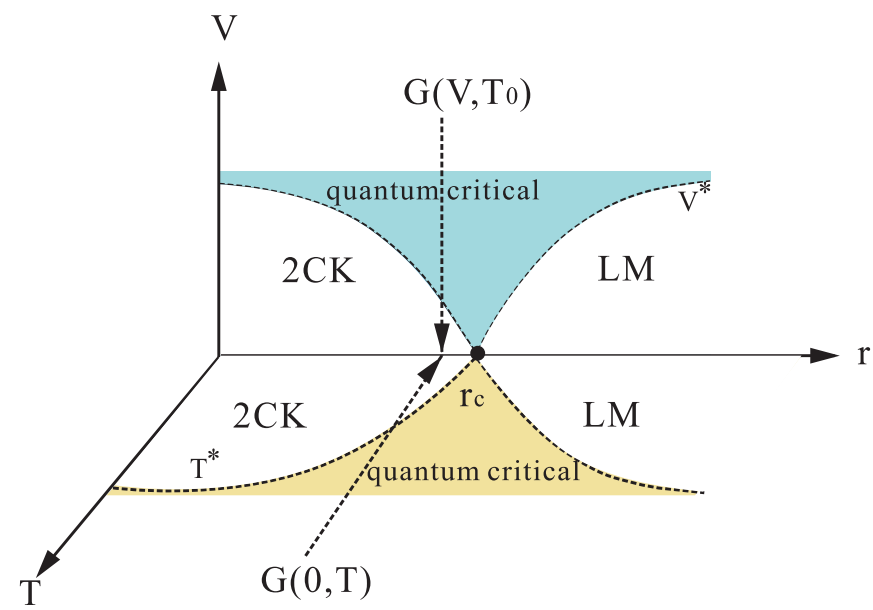

FIG. 1: Schematic 3D phase diagram of 2CK-LM quantum phase transition of the two-lead 2-channel pseudogap Anderson model as functions of voltage bias $V$, temperature $T$ and power-law exponent $r$ of the pseudogap fermion bath.

density of states (LDOS) $\rho_{c}(\omega)$ which vanishes linearly due to the Dirac spectrum: $\rho_{c}(\omega) \propto|\omega|^{r}$ with $r=1[19$ 21]. This leads to QPT from the Kondo screened phase to the unscreened local moment (LM) phase with decreasing Kondo correlation due to insufficient DOS of conduction electrons. In fact, such transitions exist in the more general framework of pseudogap Kondo (or Anderson) models with power-law exponent $0<r<1$, which have been extensively studied $[22$ 26]. However, relatively less is known on the more exotic 2CK-LM QPT in the pseudogap Anderson (Kondo) models [27, 28], in particular, when the system is subject to a non-equilibrium condition [5, 29]. 

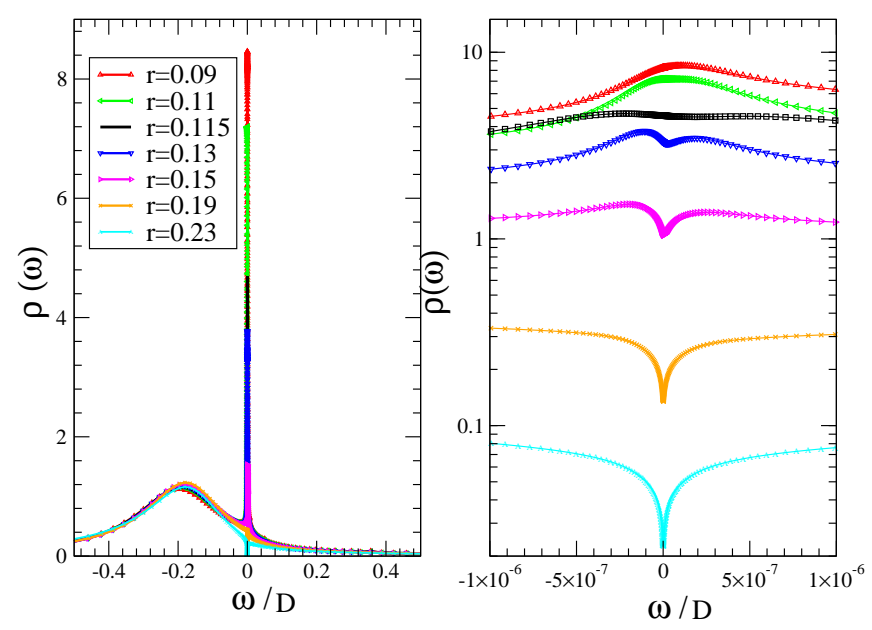

FIG. 2: The equilibrium impurity spectral function $\rho(\omega)$ (in arbitrary unit) versus energy $\omega$ (in units of the bandwidth $D$ ) with $V=0, \Gamma=0.28, \epsilon_{d}=-0.2, T=5 \times 10^{-7}$. (a). The spectral function $\rho(\omega)$ in the full energy range. For $r<r_{c}$ $\left(r_{c} \sim 0.115\right)$, the Kondo peaks are located at $\omega=0$ (Fermi energy), while for $r>r_{c}$, dips near $\omega=0$ are developed. (b). Same plots as in (a) with energies close to the peaks/dips.

Motivated by these developments, we address the 2CKLM quantum phase transition in the two-channel pseudogap Anderson model both in and out of equilibrium in a Kondo quantum dot subject to a voltage bias and finite temperature. By studying experimentally accessible steady-state transport, we search for universal scalings in both linear and non-linear conductance near criticality via a large-N method based on Non-Crossing Approximation (NCA) [8, 31 33], a reliable approach for multi-channel Kondo systems with non-Fermi liquid ground states. A fundamentally important but less addressed issue-non-equilibrium quantum criticality - is emphasized here by identifying and comparing different universal scaling behaviors between equilibrium (zero bias) and non-equilibrium (finite bias) conductances near the 2CK-LM transition [34].

\section{THE MODEL HAMILTONIAN.}

The Hamiltonian of the two-lead two-channel singleimpurity pseudogap Anderson model is formulated within the Non-Crossing Approximation (NCA) 8, 3133], a large-N approach based on the $S U(N) \times S U(M)$ generalization of the $S U(2)$ model with $N \rightarrow \infty, M \rightarrow \infty$ being the number of degenerate flavors of spins $\sigma=\uparrow$ $, \downarrow, \cdots N$ and the number of Kondo screening channels $K=1,2, \cdots M$. In the physical $S U(2)$ two-channel Kondo system, $N=M=2$. The two leads are described by a power-law vanishing density of states (DOS) at Fermi energy defined as $\rho_{c}(\omega) \sim|\omega|^{r} \Theta(D-|\omega|)$ with $0<r<1$, where $D=1$ is the bandwidth cutoff. Graphene and high $T_{c}$-cuprate superconductors are possible realizations of the pseudogap leads with $r=1$, while semiconductors with soft gaps are candidates with $0<r<1$. The Hamiltonian reads [8, 32]

$$
\begin{aligned}
H & =\sum_{\tau, \alpha, \sigma, k}\left(\epsilon_{k \sigma}-\mu_{\alpha}\right) c_{k \sigma \tau}^{\alpha \dagger} c_{k \sigma \tau}^{\alpha}+\sum_{\sigma, \tau} \epsilon_{\sigma} d_{\sigma, \tau}^{\dagger} d_{\sigma, \tau} \\
& +\frac{1}{2} U \sum_{\sigma, \sigma^{\prime}, \tau, \tau^{\prime}} n_{\sigma, \tau} n_{\sigma^{\prime}, \tau^{\prime}}\left(1-\delta_{\sigma, \sigma^{\prime}} \delta_{\tau, \tau^{\prime}}\right) \\
& +\sum_{\tau, \alpha, \sigma, k}\left(V_{k \sigma}^{\alpha} c_{k \sigma \tau}^{\alpha \dagger} d_{\sigma}+\text { h.c. }\right)
\end{aligned}
$$

where $\mu_{\alpha=L / R}= \pm V / 2$ is the chemical potential of lead $\alpha=L / R$. The operators $c_{k \sigma \tau}^{\alpha \dagger}\left(c_{k \sigma \tau}^{\alpha}\right)$ create (destroy) an electron in the leads with momentum $k$. Spin flavors are represented by $\sigma, \sigma^{\prime}=1, \cdots N$ and $\tau, \tau^{\prime}=1, \cdots M$ corresponds to $M$ independent electron reservoirs. Here, $N=M=2$. The $U$ term describes the on-site Coulomb energy on the impurity, and $V_{k \sigma}^{\alpha}$ represents the hybridization strength between the graphene electrons and the impurity.

The Hamiltonian Eq. (1) can be solved via NCA in the large- $U$ limit $U \rightarrow \infty$ with the following Hamiltonian in the pseudofermion slave-boson representation:

$$
\begin{aligned}
H & =\sum_{k \sigma \tau \alpha}\left(\epsilon_{k}-\mu_{\alpha}\right) c_{k \sigma \tau}^{\alpha \dagger} c_{k \sigma \tau}^{\alpha}+\epsilon_{d} \sum_{\sigma} f_{\sigma}^{\dagger} f_{\sigma} \\
& +\sum_{k \sigma \tau \alpha}\left(V_{k \sigma}^{\alpha}\left(f_{\sigma}^{\dagger} b_{\tau} c_{k \sigma \tau}^{\alpha}\right)+h . c\right)
\end{aligned}
$$

where the local (impurity) electron operator $d_{\sigma, \tau}^{\dagger}$ is decomposed in the pseudofermion representation as a product of pseudofermion $f_{\sigma}^{\dagger}$ and a slave-boson $b_{\tau}$ : $d_{\sigma, \tau}^{\dagger}=$ $f_{\sigma}^{\dagger} b_{\tau}$ subject to the local constraint $Q=\sum_{\sigma} f_{\sigma}^{\dagger} f_{\sigma}+$ $\sum_{\tau} b_{\tau}^{\dagger} b_{\tau}=1$ via the Lagrange multiplier $\lambda$ to ensure single occupancy on impurity.

Here, we employ the NCA to address the equilibrium and non-equilibrium transport at criticality based on Eq. (11). This approach has been known to correctly capture the non-Fermi liquid properties of the two-channel Anderson model [4, 28, 29]. Recently, it has been generalized to address the 2CK-LM crossover in non-equilibrium transport in a voltage-biased 2-channel pseudogap Anderson model with $r=1$, relevant for graphene [30]. We generalize this approach here further to the voltage-biased 2-channel pseudogap Anderson model with $0<r<1$.

Within NCA, the Green's functions for the conduction electrons $G_{\sigma, i \lambda}(t)$, pseudo-fermions $G_{f \sigma}(t)$, and the slave bosons $D(t)$ are given by [8, 32]:

$$
G_{\sigma, i \lambda}^{r}(t)=-i \theta(t)<\left\{c_{\sigma}(t), c_{\sigma}^{\dagger}(0)\right\}>_{i \lambda}
$$




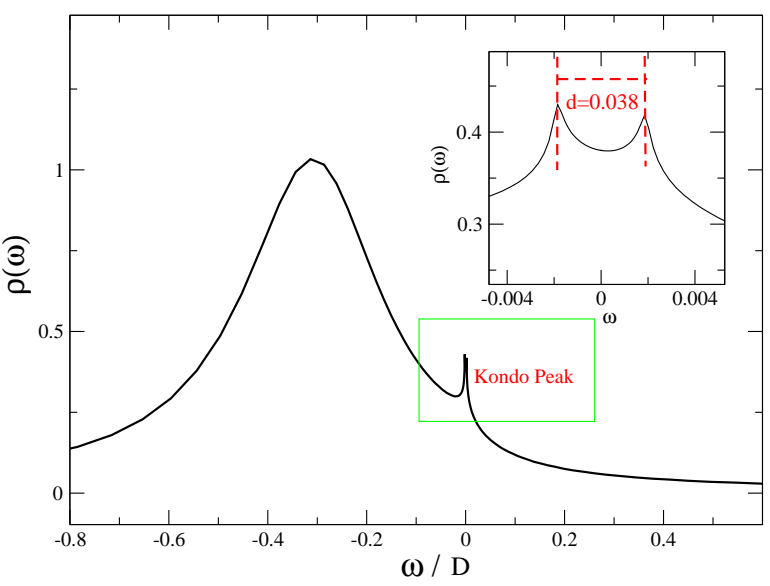

FIG. 3: The non-equilibrium impurity spectral function $\rho(\omega)$ (in arbitrary unit) with $\Gamma=0.3 D, \epsilon_{d}=-0.3 D, r=0.05$, $V=0.038 D, T=5 \times 10^{-7} D$. The Kondo peak splits into two peaks where the width of the two splited peaks equals to bias $V$ (see Inset).

$$
\begin{array}{r}
=-i \theta(t)\left[D^{>}(-t) G_{f \sigma}^{<}(t)-D^{<}(-t) G_{f \sigma}^{>}(t)\right] . \\
G_{f \sigma}^{>} \equiv-i<f_{\sigma}(t) f_{\sigma}^{\dagger}(0)>_{i \lambda}, G_{f \sigma}^{<} \equiv i<f_{\sigma}^{\dagger}(0) f_{\sigma}(t)>_{i \lambda},
\end{array}
$$

and

$$
D^{>} \equiv-i<b_{\tau}(t) b_{\tau}^{\dagger}(0)>_{i \lambda}, D^{<} \equiv i<b_{\tau}^{\dagger}(0) b_{\tau}(t)>_{i \lambda},
$$

where the notation $<$ and $>$ represents lesser and greater Green function. The lesser (greater) Green functions can be written as $[8,32]$ :

$$
\begin{aligned}
D^{>(<)}(\omega) & =D^{r}(\omega) \Pi^{>(<)}(\omega) D^{a}(\omega), \\
G_{f \sigma}^{>(<)} & =G_{f \sigma}^{r}(\omega) \Sigma_{f \sigma}^{<(>)}(\omega) G_{f \sigma}^{a},
\end{aligned}
$$

where $D^{a(r)}(\omega)$ and $G_{f \sigma}^{a(r)}(\omega)$ are advanced (retarded) Green function of boson and fermion, respectively, and the subscript $i \lambda$ refers to the enforcement of the local constraint on the impurity via Lagrange multiplier $\lambda$ when evaluating these correlation functions. The $\Pi^{>(<)}(\omega)$ and $\Sigma_{f \sigma}^{<(>)}(\omega)$ are the self-energies of slave-boson and pseudofermion greater (lesser) Green functions, respectively. The NCA expressions for the lesser self-energy of the pseudofermion, $G_{f \sigma}^{<}(\omega)=\Sigma^{<}(\omega)\left|G_{f \sigma}^{r}(\omega)\right|^{2}$, and slaveboson, $D^{<}(\omega)=\Pi^{<}(\omega)\left|D^{r}(\omega)\right|^{2}$, are [8, 30, 32] :

$\Sigma_{f \sigma}^{<}(\omega)=\frac{2}{\pi} \sum_{\alpha} \int d \epsilon \Gamma_{\alpha}\left(\omega-\epsilon-\mu_{\alpha}\right) f\left(\omega-\epsilon-\mu_{\alpha}\right) D^{<}(\epsilon)$

$\Pi^{<}(\omega)=\frac{2}{\pi} \sum_{\alpha} \int d \epsilon \Gamma_{\alpha}\left(\epsilon-\omega-\mu_{\alpha}\right) f\left(\omega-\epsilon+\mu_{\alpha}\right) G_{f \sigma}^{<}(\epsilon)$.

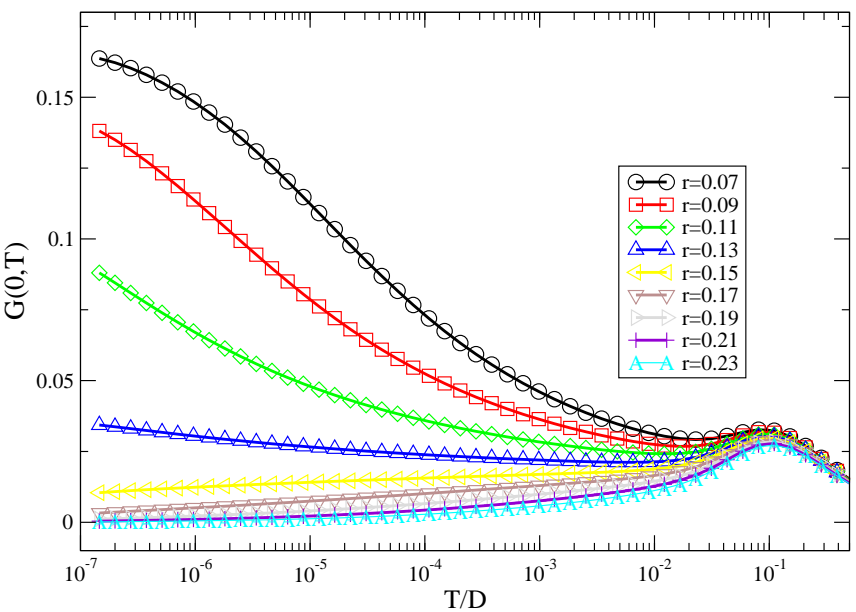

FIG. 4: The equilibrium (linear) conductance $G(0, T)$ (in unit of $2 e / \hbar$ ) versus temperature $T$ (in unit of $D=1$ ) for various $r$ with $\Gamma=0.3 D, \epsilon_{d}=-0.3 D$.

Here, $\Gamma_{\alpha}(\omega) \equiv \Gamma_{\alpha} \rho_{c, \alpha}(\omega)$ with $\Gamma_{\alpha}=\pi\left|V_{k \sigma}^{\alpha}\right|^{2}$ with $\rho_{c, \alpha}(\omega)=-\frac{1}{\pi} \operatorname{Im} G_{c}(\omega)=\frac{r+1}{2 D^{r+1}}|\omega|^{r} \theta\left(D-\left|\omega-\mu_{\alpha}\right|\right)$ and $D=1$ being the bandwidth of the conduction bath, and $f(\omega)=\frac{1}{1+e^{\beta \omega}}$ is the Fermi function.

The relation between greater Green function and retarded Green function are, $D^{>}(w)=2 i \operatorname{Im} D^{r}(w), G_{f \sigma}^{>}=$ $2 i \operatorname{Im} G_{f \sigma}^{r}$, where the self-energies, $\Pi^{>}(w)=2 i \operatorname{Im} \Pi^{r}(w)$ and $\Sigma_{f \sigma}^{>}(w)=2 i \operatorname{Im} \Sigma_{f \sigma}^{r}(w)$. The NCA expressions for the self energies of retarded Greens functions for pseudofermion $G_{f \sigma}^{r}(\omega)=\left[\omega-\epsilon_{d}-\Sigma^{r}(\omega)\right]^{-1}$ and for slave-boson $D^{r}(\omega)=\left[\omega-\Pi^{r}(\omega)\right]^{-1}$ are given by $\left.8, \underline{80}, \underline{32}\right]$ :

$\Sigma^{r}(\omega)=\frac{2}{\pi} \sum_{\alpha} \int d \epsilon \Gamma_{\alpha}\left(\omega-\epsilon-\mu_{\alpha}\right) f\left(\epsilon-\omega-\mu_{\alpha}\right) D^{r}(\epsilon)$,

$\Pi^{r}(\omega)=\frac{2}{\pi} \sum_{\alpha} \int d \epsilon \Gamma_{\alpha}\left(\epsilon-\omega-\mu_{\alpha}\right) f\left(\epsilon-\omega-\mu_{\alpha}\right) G_{f \sigma}^{r}(\epsilon)$.

The physical impurity spectral function, $\rho_{\sigma}(\omega, V)$, is obtained via the convolution of pseudo-fermion and slaveboson Greens function based on Eqs. (77), (8), (99), and (10) as [30]:

$$
\begin{array}{r}
\rho(\omega, V)=\frac{i}{2 \pi^{2} Z} \int d \epsilon\left[\operatorname{Im} D^{r}(\epsilon) G^{<}(\omega+\epsilon)-\right. \\
\left.D^{<}(\epsilon) \operatorname{Im} G^{r}(\omega+\epsilon)\right] .
\end{array}
$$

The normalization factor $Z=\frac{i}{2 \pi} \int d \omega\left[M \times D^{<}(\omega)-N \times\right.$ $\left.G^{<}(\omega)\right]$ enforces the constraint, $\langle Q\rangle=1$ with $M=$ (7) $N=2$ here. The current going through the impurity therefore reads [30]:

$$
\begin{aligned}
I(V, T) & =\frac{2 e}{\hbar} \int d \omega \frac{2 \Gamma_{L}(\omega) \Gamma_{R}(\omega)}{\Gamma_{L}(\omega)+\Gamma_{R}(\omega)} \rho(\omega, V, T) \\
& \times[f(\omega+e V / 2)-f(\omega-e V / 2)] .
\end{aligned}
$$




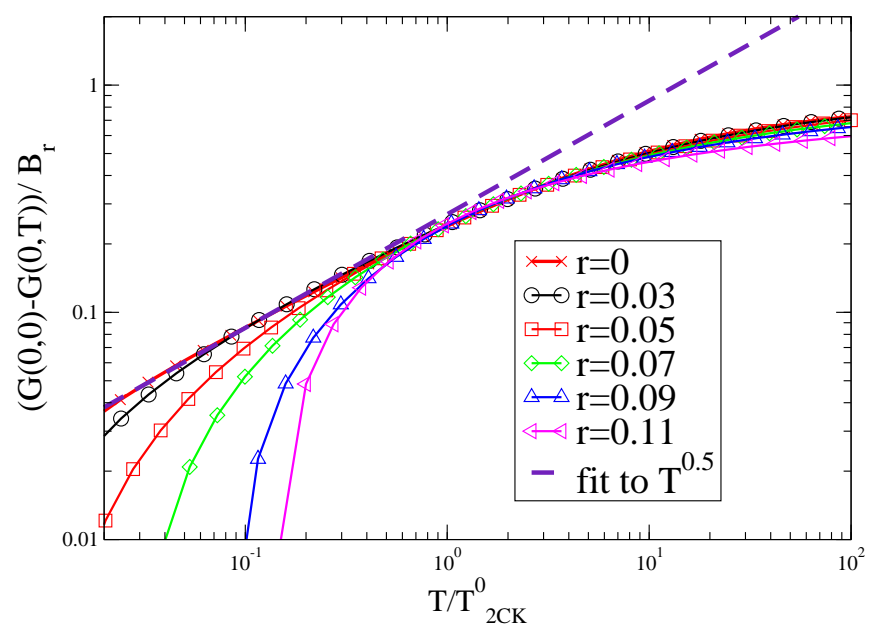

FIG. 5: The $\sqrt{T}$ scaling of equilibrium (linear) conductance $(G(0,0)-G(0, T)) / B_{r}$ (in unit of $2 e / \hbar$ ) versus $T / T_{2 C K}^{0}$ for various $r$ with $\Gamma=0.28 D, \epsilon_{d}=-0.2 D$ with $T_{2 C K}^{0}$ being the two-channel Kondo temperature for $r=0$. Here, $B_{r}$ are non-universal functions of $r$. The dashed line is a fit to $\sqrt{T}$ behavior.

where $\Gamma_{\alpha}(\omega)=\Gamma\left(\omega-\mu_{\alpha}\right)$ with $\alpha=L, R$. The equilibrium (linear) conductance is directly obtained via

$$
\begin{aligned}
G(0, T) & =\frac{2 e^{2}}{\hbar} \int d \omega \frac{2 \Gamma_{L}(\omega) \Gamma_{R}(\omega)}{\Gamma_{L}(\omega)+\Gamma_{R}(\omega)}\left(-\frac{\partial f(\omega)}{\partial \omega}\right) \\
& \times \rho(\omega, V=0) .
\end{aligned}
$$

And the nonlinear conductance $G(V)$ is given by $\frac{d I(V)}{d V}$. Eqs.(6)-(10) form a self-consistent set of Dyson's equations within NCA. We solve these equations selfconsistently and evaluate Eqs.(11)-(13) based on these solutions.

\section{RESULTS.}

\section{A. Quantum critical point at 2CK-LM phase transition: impurity spectral function}

The existence of a quantum critical point (QCP) separating 2CK for $r<r_{c}$ from the LM for $r>r_{c}$ phases exists in the PSG Anderson model has been studied extensively [28, 35]. The generic phase diagram is shown as in Fig. 1 We focus here on the transport properties for the two-lead setup near criticality both in and out of equilibrium, especially on the distinct non-equilibrium quantum critical properties (see Fig. 1). In equilibrium $\left(\mu_{\alpha}=0\right)$, the 2CK-LM phase transition is studied here by tuning $r$ of the pseudogap power-law DOS of the leads with fixed hybridization parameter $\Gamma_{\alpha}$ and the impurity energy $\epsilon_{d}$. The value of $r_{c}$ is extracted from the local impurity spectral function $\rho_{\sigma}(\omega, V=0)$ via solving the self-consistent Dyson's equations. Since $\epsilon_{d} \neq-U / 2$, the

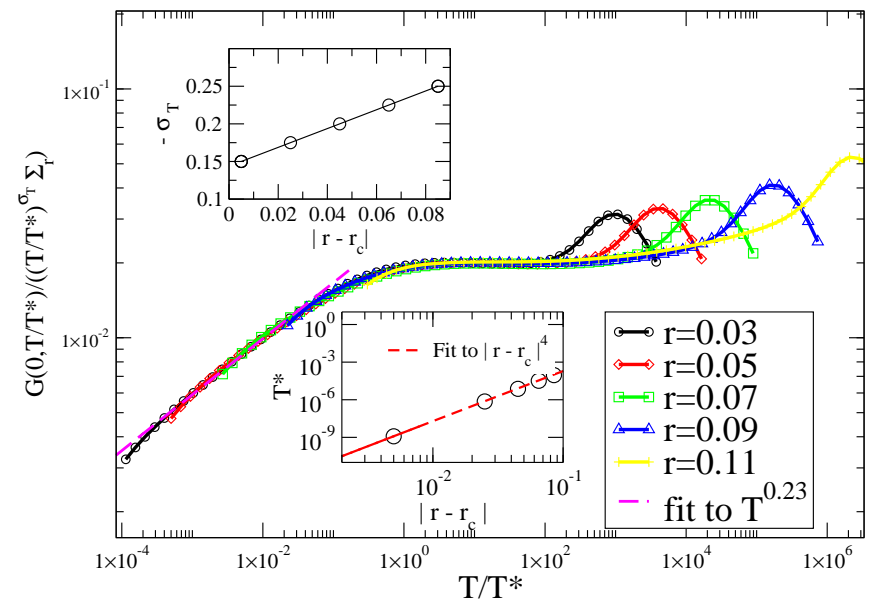

FIG. 6: Universal scaling in linear conductance $G\left(0, T / T^{*}\right)$ (in unit of $2 e / \hbar$ and normalized to $\Sigma_{r}\left(T / T^{*}\right)^{\sigma_{T}}$ ) as a function of temperature $T / T^{*}$ near 2CL-LM quantum phase transition for various values of $r$. Parameters are the same as in Fig. 4. Here, $T^{*}, \sigma_{T}$, and $\Sigma_{r}$ are defined in the text. The magenta dashed line is a fit to $T^{0.23}$. Top Inset: The power-law exponent $\sigma_{T}$ in linear conductance $G_{Q C P}(T)$ close to criticality as a function of $\left|r-r_{c}\right|$. Solid line is a fit to a linear relation: $\sigma_{T}=\beta_{T}-\alpha_{T}\left|r-r_{c}\right|$ with $\beta_{T} \approx-0.145, \alpha_{T} \approx 1.25$. Bottom Inset: Crossover temperature $T^{*}$ versus $\left|r-r_{c}\right|$. The red dashed line is a fit to $\left|r-r_{c}\right|^{4}$.

2CK pseudogap Anderson model considered here shows particle-hole (ph) asymmetry, giving rise to an overall asymmetric impurity spectral function with respect to the Fermi energy (see Fig. 22). For $r<r_{c}, \rho_{\sigma}(\omega)$ shows a non-Lorentzian Kondo peak, a characteristic of the nonFermi liquid 2CK state [4, [8]. In fact, $\rho_{\sigma}(\omega)$ exhibits a power-law singularity near $\omega=0$ with an exponent being $r: \rho_{\sigma}(\omega) \sim \omega^{-r}[28]$. With increasing $r$, the Kondo peak gets narrower with reduced spectral weight. For $r \leq r_{c}$, however, the Kondo peak splits into two, and the ground state is in the LM phase. The critical value $r_{c} \approx 0.115$ for $\Gamma_{\alpha} \sim 0.3 D, \epsilon_{d} \sim-0.2 D$ (see Fig. 2). The spectral weight of the Kondo peaks gets further suppressed with increasing $r$ until it completely disappears. At a finite bias voltage for $r<r_{c}$, the impurity local DOS shows splited Kondo peaks at $\omega= \pm V / 2$ (see Fig. (3)). Note that the non-zero local DOS of $\rho_{\sigma}(\omega=0)$ for $r>r_{c}$ is due to the limitation of the lowest temperature $T_{0} \sim 5 \times 10^{-7} D$ we can reach numerically. As $T \rightarrow 0$ and for $r \geq r_{c}$, we expect a complete dip developed in local DOS such that $\rho_{\sigma}(\omega=0) \sim 0$. 


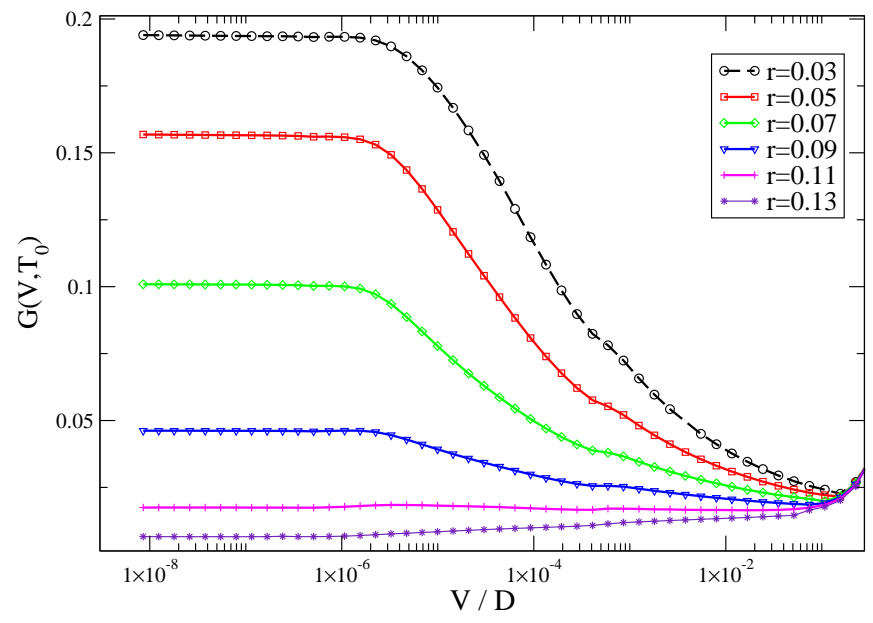

FIG. 7: The non-equilibrium (non-linear) conductance $G\left(V, T_{0}\right)$ (in unit of $2 e / \hbar$ ) versus bias voltage $V$ (in unit of $D=1$ ) for various $r$ with $\Gamma=0.3 D, \epsilon_{d}=-0.3 D$.

\section{B. Universal scaling in linear (equilibrium) conductance near criticality}

More clear signatures of the 2CK-LM quantum phase transition can be obtained via linear and non-linear conductances. First, we analyze the linear (equilibrium) conductance at finite temperatures but zero bias voltage $G(V=0, T)$. Fig. 田 shows $G(0, T)$ for different $r$ with $\Gamma=0.28 D, \mu_{0}=0, \epsilon_{d}=-0.2 D$, and $D=1$. For $r=0$ it is well known that $G(0, T)$ follows the $2 \mathrm{CK}$ scaling function[8]: $G(0, T)-G(0,0)=B_{r} \sqrt{T / T_{2 C K}^{0}}$ for $T<T_{2 C K}^{0}$ with $T_{2 C K}^{0} \sim 3 \times 10^{-5} D$ being the 2 CK Kondo temperature at $r=0$ and $B_{r}$ being a non-universal constant. Here, we set $G(0,0) \simeq G\left(0, T_{0}=5 \times 10^{-7} D\right)$. For $0<r<r_{c}$, however, the 2CK $\sqrt{T}$ scaling in $G(0,0)-G(0, T)$ ceases to exist. As shown in Fig. [4 the $\sqrt{T}$ behavior in $G(0,0)-G(0, T)$ is clear for $r=0$, but it deviates from $\sqrt{T}$ more with increasing $r<r_{c}$. We propose that this deviation from the conventional 2CK behavior for $T<T_{2 C K}$ for $0<r<r_{c}$ can be due to the following two scenarios: (i). the emergence of distinct universal power-law scaling behaviors when the system approaches the 2CK-LM quantum critical regime for $T>T^{*}$ with $T^{*}$ being the crossover energy scale above which quantum critical behaviors are observed, and (ii). the existence of a distinct $2 \mathrm{CK}$ scaling form other than $\sqrt{T}$ in conductance in low temperature regime $T<T_{2 C K}$ for $0<r<r_{c}$ with $T_{2 C K}$ being the two-channel Kondo temperature for $0<r<r_{c}$. As indicated in Fig. 5 the generic $2 \mathrm{CK}$ behaviors for $0<r<r_{c}$ can not be manifested in $G(0,0)-G(0, T)$ as an universal power-law in $T$ as it does for $r=0$ case. Therefore, instead of analyzing $G(0,0)-G(0, T)$, we therefore address the above two scenarios below via trying the possible scaling behaviors of $G(T)$ itself.
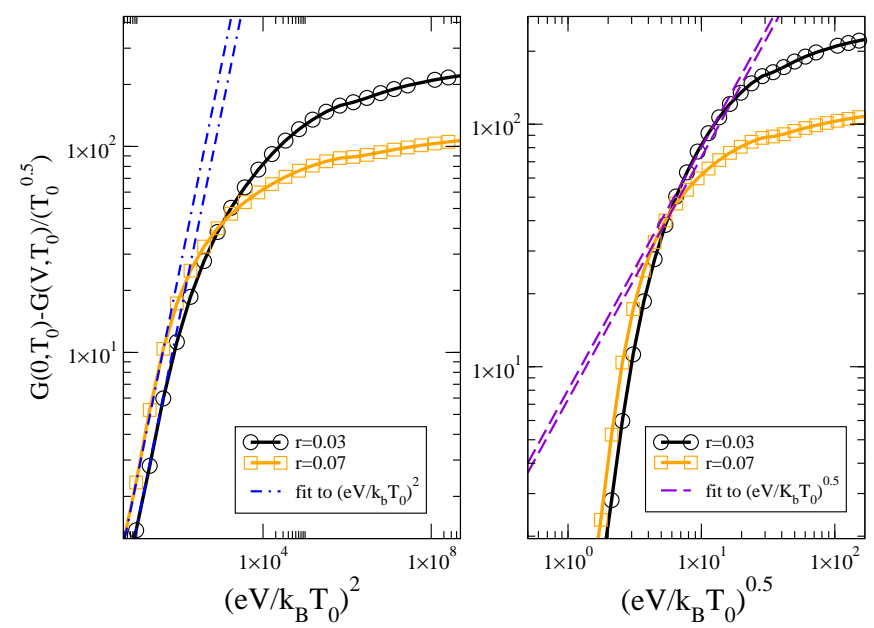

FIG. 8: The 2CK scaling of the non-equilibrium (non-linear) conductance $\left(G\left(0, T_{0}\right)-G\left(V, T_{0}\right)\right) / T_{0}^{0.5}$ (in unit of $2 e / \hbar$ ) versus (a) $\left(e V / k_{B} T\right)^{2}$ and (b) $\left(e V / k_{B} T\right)^{0.5}$ for various $r$ with $\Gamma=0.3 D, \epsilon_{d}=-0.3 D$.

First, as $r \rightarrow r_{c}$, the existence of a quantum critical regime for $T>T^{*}$ requires the divergence of the correlation length $\xi$ in a power-law fashion: $\xi \propto\left|r-r_{c}\right|^{-\nu} \rightarrow$ $\infty[1,2]$. As a result, all thermaldynamical observables, including conductances, are expected to exhibit universal scaling properties. As shown in Fig. 6, we indeed find numerically that the linear conductance $G_{Q C P}(0, T)$ shows an universal power-law in $T$ near criticality within a temperature range of (approximately) $5 \times 10^{-7} D<$ $T<5 \times 10^{-4} D$ as:

$$
G_{Q C P}(0, T) \propto T^{\sigma_{T}}=T^{\beta_{T}-\alpha_{T}\left|r-r_{c}\right|},
$$

where the exponent $\sigma_{T}$ exhibits a linear relation to $\left|r-r_{c}\right|$ with $\beta_{T} \approx-0.145$ and $\alpha_{T} \approx 1.25$ being non-universal constant pre-factors dependent on $\Gamma$ and $\epsilon_{d}$, and $T^{\sigma_{T}}=$ $T^{\beta_{T}}$ as $r=r_{c} \sim 0.115$. Based on the above analysis, we divide $G(0, T)$ by the power-law function $T^{\sigma_{T}}$, gives:

$$
\begin{aligned}
\widetilde{G}(0, T) & =G(0, T) / T^{\sigma_{T}}=\frac{G(0, T)}{T^{\beta_{T}-\alpha_{T}\left|r-r_{c}\right|}}, \\
\widetilde{G}_{Q C P}(0, T) & =G_{Q C P}(0, T) / T^{\sigma_{T}}
\end{aligned}
$$

where $\widetilde{G}_{Q C P}(0, T)$ in the quantum critical region becomes a constant: $\widetilde{G}_{Q C P}(0, T) \sim G_{Q C P}^{0}$. The universal scaling function $\bar{G}\left(0, \frac{T}{T^{*}}\right)$ in linear conductance is obtained by rescaling $\widetilde{G}(0, T)$ by a non-universal factor $\Sigma_{r}$ and $T$ by the crossover energy scale $T^{*}$ (see Fig. 6):

$$
\bar{G}\left(0, \frac{T}{T^{*}}\right) \equiv \frac{\tilde{G}\left(0, \frac{T}{T^{*}}\right) / \Sigma_{r}}{\left(T / T^{*}\right)^{\beta_{T}-\alpha_{T}\left|r-r_{c}\right|}},
$$

where $T^{*}$ is proportional to the inverse of correlation length $1 / \xi_{T} \propto\left|r-r_{c}\right|^{\nu_{T}}$, vanishing in a power-law of 


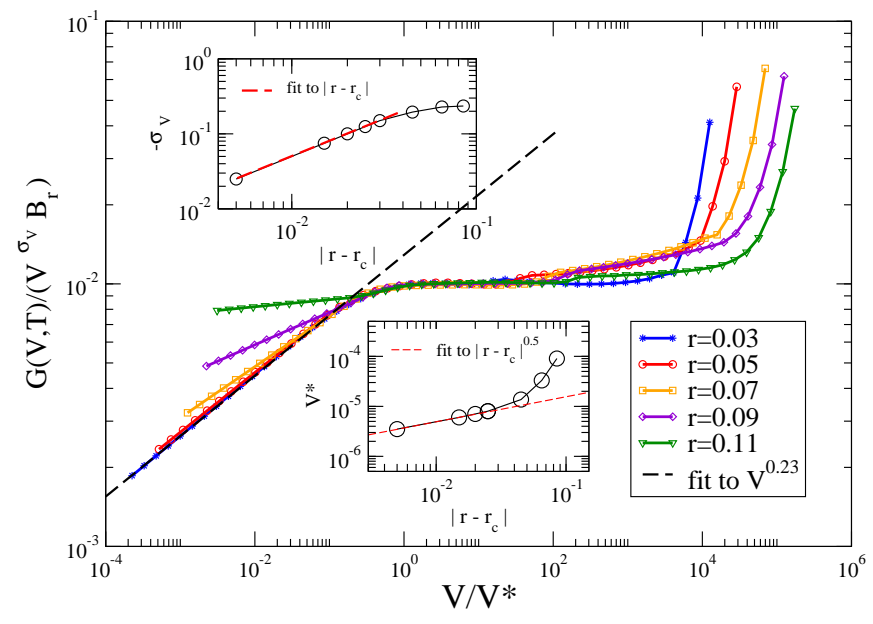

FIG. 9: Universal scaling in non-linear conductance $G\left(V, T_{0}\right) /\left(V_{V}^{\sigma} B_{r}\right)$ (in unit of $2 e / \hbar$ ) as a function of temperature $V / V^{*}$ near 2CL-LM quantum phase transition for various values of $r$. Parameters are the same as in Fig. 4 . Here, $V^{*}$, $\sigma_{T}$, and $B_{r}$ are defined in the text. Black dashed line is a power-law fit to the conductance in the $2 \mathrm{CK}$ regime. Top Inset: The power-law exponent $\sigma_{V}$ in non-linear conductance $G_{Q C P}\left(V, T_{0}\right)$ close to criticality as a function of $\left|r-r_{c}\right|$. Solid line is a fit to a linear relation: $\sigma_{V}=\beta_{V}-\alpha_{V}\left|r-r_{c}\right|$ with $\beta_{V} \approx 0, \alpha_{V} \approx 2.5$. Bottom Inset: Crossover temperature $V^{*}$ versus $\left|r-r_{c}\right|$. The red dashed line is a fit to $\left|r-r_{c}\right|^{0.5}$.

$\left|r-r_{c}\right|$ with the exponent $\nu_{T}$ being the correlation length exponent:

$$
T^{*} \propto \frac{1}{\xi_{T}}=D\left|r-r_{c}\right|^{\nu_{T}}
$$

with $\nu_{T}$ being the correlation length exponent corresponding to the power-law exponent of crossover scale $T^{*}$ versus $\left|r-r_{c}\right|$. We find $\nu_{T} \sim 4$ here. (see Inset of Fig. 6). As $r \rightarrow r_{c}$ from below, we find a perfect data collapse in $\bar{G}\left(0, \frac{T}{T^{*}}\right) \approx$ const. for $1<T / T^{*}<10^{3}$, indicating the range of quantum critical region. Surprisingly, at low temperatures $T<T_{2 C K} \approx 0.1 T^{*}$ where the system is governed by the 2 CK phase, we find the above function $\bar{G}\left(0, \frac{T}{T^{*}}\right)$ exhibits as a distinct universal function power-law scaling function (see Fig. 6):

$$
\bar{G}\left(0, \frac{T}{T^{*}}\right)_{2 C K} \propto\left(\frac{T}{T_{2 C K}}\right)^{\sigma_{2 C K}^{T}}
$$

with $\sigma_{2 C K}^{T} \sim 0.23$ for the parameters we set (or equivalently $\left.G\left(\frac{T}{T_{2 C K}}\right) \propto\left(\frac{T}{T_{2 C K}}\right)^{\sigma_{2 C K}^{T}}+\sigma_{T}\right)$. We may therefore regard this low temperature behavior in $G(0, T)$ as the distinct $2 \mathrm{CK}$ scaling in equilibrium (linear) conductance for the pseudogap 2-channel Anderson model.

\section{B. Universal scaling in non-linear (non-equilibrium) conductance near criticality}

We now add the bias voltage in the leads and study the scalings in non-equilibrium conductance near 2CKLM quantum critical point. It is generally expected that the scaling behaviors of non-linear conductance near criticality are distinct from that in equilibrium [5]. The nonlinear conductance $G\left(V, T_{0}\right)$ is obtained at a fixed low temperature $T_{0}=5 \times 10^{-7}$, symmetrical hybrizidation $\Gamma_{L}=\Gamma_{R}=0.3 D$, and $\epsilon_{d}=-0.3 D$. As shown in Fig. 7 . the non-linear conductance $G\left(V, T_{0}\right)$ for each value of $r$ saturates as $V \rightarrow 0$, while a small "kink"-like minima in conductance is developed at $e V \approx k_{B} T$. For $r=0$ and $T<T_{2 C K}^{0}$, it follows the well-known $2 \mathrm{CK}$ scaling $[8]$ : $G\left(0, T_{0}\right)-G\left(V, T_{0}\right)=\Sigma_{r} T^{\frac{1}{2}} H\left(\frac{e V}{k_{B} T_{0}}\right)$, where the universal function $H\left(\frac{e V}{k_{B} T_{0}}\right)$ behaves as $\left(\frac{e V}{k_{B} T_{0}}\right)^{2}$ for $e V \leq k_{B} T_{0}$, and $\left(\frac{e V}{k_{B} T_{0}}\right)^{\frac{1}{2}}$ for $e V \gg k_{B} T_{0}$. However, for $0<r<r_{c}$ as the system gets closer to criticality, while $\left(\frac{e V}{k_{B} T_{0}}\right)^{2}$ behavior remains in $G\left(0, T_{0}\right)-G\left(V, T_{0}\right)$ for $\mathrm{eV} \sim k_{B} T_{0}$, more deviations from the $2 \mathrm{CK}\left(\frac{e V}{k_{B} T_{0}}\right)^{\frac{1}{2}}$ behavior are observed for $k_{B} T_{0}<e V<k_{B} T_{2 C K}$. Furthermore, similar to the case in our analysis for equilibrium conductance, as indicated in Fig. 8, the universal 2CK behavior in non-linear conductance for $0<r<r_{c}$ in general are not manifested in $G\left(0, T_{0}\right)-G\left(V, T_{0}\right)$ in the power-law fashion as it is for $r=0$ case. We therefore analyze the universal scaling properties based on $G\left(V, T_{0}\right)$ itself rather than $G\left(0, T_{0}\right)-G\left(V, T_{0}\right)$. We perform the similar analysis here to that in equilibrium. As $r \rightarrow r_{c}$, we find $G\left(V, T_{0}\right)$ shows a power-law dependence on $V$ in the quantum critical regime approximately $10^{-4}<V / D<10^{-6}$, defined as $G_{Q C P}\left(V, T_{0}\right)$ with power-law exponent linear in $\left|r-r_{c}\right|$ :

$$
G_{Q C P}\left(V, T_{0}\right) \propto V^{\sigma_{V}}=V^{\beta_{V}-\alpha_{V}\left|r-r_{c}\right|}
$$

with $\beta_{V}, \alpha_{V}$ being non-universal prefactors dependent on $\Gamma$ and $\epsilon_{d}$. At criticality $r=r_{c}, \sigma_{V} \sim 0$, yielding a constant non-linear conductance: $\left.G_{Q C P}\left(V, T_{0}\right)\right|_{r_{c}} \sim$ const.. We further perform a re-scaling on $G\left(V, T_{0}\right)$ as:

$$
\tilde{G}\left(V, T_{0}\right) \equiv \frac{G\left(V, T_{0}\right)}{V^{\sigma_{V}}}=\frac{G\left(V, T_{0}\right)}{V^{\beta_{V}-\alpha_{V}\left|r-r_{c}\right|}}
$$

where $\tilde{G}\left(V, T_{0}\right)$ becomes a constant in the critical regime. We may further define the universal scaling function $\bar{G}\left(\frac{V}{V^{*}}, T_{0}\right)$ for non-linear conductance via the following re-scalings: $V \rightarrow V / V^{*}, \tilde{G}\left(V, T_{0}\right) \rightarrow \tilde{G}\left(V, T_{0}\right) / \Sigma_{r}$ (see Fig. (9):

$$
\bar{G}\left(\frac{V}{V^{*}}, T_{0}\right)=\frac{G\left(\frac{V}{V^{*}}, T_{0}\right) / \Sigma_{r}}{\left(\frac{V}{V^{*}}\right)^{\beta_{V}-\alpha_{V}\left|r-r_{c}\right|}}
$$

where $V^{*}$ is the crossover energy scale, and $\Sigma_{r}$ a nonuniversal constant pre-factor. Here, we find the exponent $\sigma_{V}$ depends linearly on $\left|r-r_{c}\right|$ with $\beta_{V} \approx 0, \alpha_{V} \approx 2.5$ 
by the best fit of the data (see Inset of Fig. 9), and the crossover scale $V^{*}$ is inversely proportional to the correlation length $\xi_{V}$ with a power-law dependence on the distance to criticality: $V^{*} \propto \frac{1}{\xi_{V}} \propto\left|r-r_{c}\right|^{\nu_{V}}$ with $\nu_{V} \sim 0.5$ (see Inset of Fig 9). Note that these critical exponents out of equilibrium are distinct from those in equilibrium, and can be considered as characteristics of non-equilibrium quantum criticality. The distinction between equilibrium and nonequilibrium quantum critical properties is expected due to the different role played by the temperature and voltage bias near criticality, leading to different behaviors in decoherence rate $\Gamma^{s}$ (the broadening of impurity DOS) in equilibrium $\Gamma_{T}^{s}$ versus out of equilibrium $\Gamma_{V}^{s}[\underline{5}, 36]$.

For $T_{0}<V<V^{*}$, the system approaches $2 \mathrm{CK}$ state at a characteristic energy scale $V \sim V_{2 C K} \approx 0.1 V^{*}$ below which an universal power-law scaling is observed (see Fig. 9):

$$
\bar{G}\left(\frac{V}{V^{*}}, T_{0}\right)_{2 C K} \propto\left(\frac{V}{V *}\right)^{\sigma_{2 C K}^{V}}
$$

where the exponent $\sigma_{2 C K}^{V} \sim 0.23$. In the low bias limit $V<T_{0}$, conductance reaches a constant equilibrium value $G\left(0, T_{0}\right)$ and therefore deviates from the $2 \mathrm{CK}$ scaling (see Fig. 9).

Note that we find Eq. (22) for non-equilibrium conductance to exhibit the same form as that in equilibrium in Eq. (18) with the same exponent $\left(\sigma_{2 C K}^{T}=\sigma_{2 C K}^{V} \sim 0.23\right)$. Further studies are required to clarify if this relation holds in general. Though the exact value $(0.23)$ of the above exponents depend in general on physical parameters, such as: $\epsilon_{d}$ and $\Gamma$, Eqs. (18) and (22) correctly reproduce the well-known $\sqrt{V}$ and $\sqrt{T}$ behaviors for $r=0$ in the $2 \mathrm{CK}$ regime in $G\left(0, T_{0}\right)-G\left(V, T_{0}\right)$ and $G\left(0, T_{0}\right)-G(0, T)$, respectively.

\section{CONCLUSIONS.}

In conclusions, we have studied quantum phase transitions in and out of equilibrium between two-channel Kondo and local moment phases in the two-channel pseudogap Anderson model where the conduction leads show a power-law vanishing density of states with exponent $0<r<1$. Via Non-Crossing Approximation (NCA), we solved self-consistently for the impurity Green's function, and therefore determined the linear and non-linear conductances. The 2CK-LM quantum criticality is reached by varying the power-law exponent $r$ of the pseudogap conduction bath with fixed lead-dot hybridizations and chemical potentials. The linear $G(V=0, T)$ and nonlinear $G\left(V, T_{0}\right)\left(T_{0} \sim 5 \times 10^{-7} \mathrm{D}\right)$ conductances show distinct universal power-law scalings near criticality with different critical exponents. Furthermore, in the 2CK regime $T, V<T_{2 C K}$, we also find different characteristic power-law scalings in $G(T)$ and $G\left(V, T_{0}\right)$ compared to the well-known $\sqrt{T}, \sqrt{V}$ scalings for $G(0,0)-G(0, T)$ and $G(V, 0)-G\left(V, T_{0}\right)$, respectively. Our results provide further insights on two-channel Kondo physics and on the non-equilibrium quantum criticality in nano-systems. Further analytic and numerical investigations are needed to address issues on the mechanism behind the different scalings between equilibrium and nonequilibrium conductances.

We thank S. Kirchner and K. Ingersent for useful discussions. C.H. C. acknowledges the NSC grant No.98-2112-M-009-010-MY3, No.101-2628-M-009001-MY3, the NCTS, the MOE-ATU program of Taiwan R.O.C.

[1] S. Sachdev, Quantum phase transitions, Cambridge University press (2000); S. L. Sondhi, S. M. Girvin, J. P. Carini, and D. Shahar, Rev. Mod. Phys. 69, 315 (1987).

[2] M. Vojta, Phil. Mag. 86, 1807 (2006).

[3] L. Kouwenhoven and L. Glazman, Physics World 14, 33 (2001); D. Goldhaber-Gordon et al., Nature 391, 156 (1998); W. G. van der Wiel et al., Science 289, 2105 (2000); L.I. Glazman and M.E. Raikh, Sov. Phys. JETP Lett. 47, 452 (1988); T.K. Ng, P.A. Lee, Phys. Rev. Lett. 61, 1768 (1988).

[4] A.C. Hewson, The Kondo Problem to Heavy Fermions (Cambridge University Press, Cambridge, UK, 1997).

[5] Chung-Hou Chung, Karyn Le Hur, Matthias Vojta, Peter Wlfle, Phys. Rev. Lett. 102, 216803 (2009); Chung-Hou Chung, Karyn Le Hur, Gleb Finkelstein, Matthias Vojta, Peter Woelfle, Phys. Rev. B 87, 245310 (2013); ChungHou Chung, Kenneth Yi-Jie Zhang, Phys. Rev. B 85, 195106 (2012);S. Kirchner and Q. Si, Phys. Rev. Lett. 103, 206401 (2009).

[6] P. Nozieres, A. Blandin, J. Phys. (Paris) 41, 193 (1980).

[7] I. Affleck, and A.W.W. Ludwig, Nucl. Phys. B 360, 641 (1990); I. Affleck and A.W.W. Ludwig, Phys. Rev. Lett. 67, 161 (1991).

[8] M. H. Hettler, J. Kroha, and S. Hershfield, Phys. Rev. Lett. 73, 1968 (1994); M.H. Hettler, J. Kroha, and S. Hershfield, Phys. Rev. B 58, 5649 (1998).

[9] P. Fendley, F. Lesage, and H. Saleur, J. Stat. Phys. 79, Nos. 5/6, 799 (1995).

[10] Paul Fendley, Matthew P.A. Fisher, Chetan Nayak, Phys. Rev. B 75, 045317 (2007); Gregory A. Fiete, Waheb Bishara, Chetan Nayak, Phys. Rev. Lett. 101, 176801 (2008).

[11] Eran Sela, Andrew K. Mitchell, Lars Fritz, Phys. Rev. Lett. 106, 147202 (2011); Andrew K. Mitchell, Eran Sela, and David E. Logan, Phys. Rev. Lett. 108, 086405 (2012).

[12] N. Andrei and C. Destri, Phys. Rev. Lett. 52, 364 (1984), P. B. Wiegmann and A. M. Tsvelik, Z. Phys. B 54, 201 (1985).

[13] I. Affleck and A. W. W. Ludwig, Nucl. Phys. B 352, 849 (1991); ibid. 360, 641 (1991). A.W.W. Ludwig, I. Affleck, Phys. Rev. Lett. 67, 3160 (1991), E. Sela, A.K. Mitchell, L. Fritz, Phys. Rev. Lett. 106, 147202 (2011).

[14] V.J. Emery, S. Kivelson, Phys. Rev. B 46, 10812 (1992); M. Fabrizio, A.O. Gogolin, Phys. Rev. B 51, 17827 
(1995).

[15] A. K. Mitchell, E. Sela, D. E. Logan, Phys. Rev. Lett. 108, 086405 (2012).

[16] R.M. Potok, I.G. Rau, H. Shtrikman, Y. Oreg, and Goldhaber-Gordon, Nature London 446, 167 (2007).

[17] D. L. Cox and A. Zawadowski,Adv. Phys. 47, 599 (1998).

[18] T. Chichorek et al., Phys. Rev. Lett. 94, 236603 (2005).

[19] K. Sengupta and G. Baskaran, Phys. Rev. B 77, 045417 (2008).

[20] Matthias Vojta, Lars Fritz and Ralf Bulla, Europ. Phys. Lett. 90, 27006 (2010).

[21] L. S. Mattos et al., (un-published); L.S. Mattos, "Correlated electrons probed by scanning tunneling microscopy", PhD. thesis, Stanford University (2009).

[22] David Withoff and Eduardo Fradkin, Phys. Rev. Lett. 64, 1835 (1990).

[23] C. Gonzalez-Buxton and K. Ingersent, Phys. Rev. B 57, 14254 (1998).

[24] K. Ingersent and Q. Si, Phys. Rev. Lett. 89, 076403 (2002).

[25] Matthew Glossop et al., Phys. Rev. Lett. 107, 076404 (2011).

[26] Lars Fritz and Matthias Vojta, Phys. Rev. B 70, 214427 (2004).
[27] I. Schneider, L. Fritz, F. B. Anders, A. Benlagra, M. Vojta, Phys. Rev. B 84, 125139 (2011).

[28] F. Zamani, T. Chowdhury, P. Ribeiro, K. Ingersent, and S. Kirchner, pss in print (2013) and arXiv:1211.4450.

[29] S. Kirchner, F. Zamani, E. Munoz, chapter contributed to "New Materials for Thermoelectric Applications: Theory and Experiment", Springer Series: NATO Science for Peace and Security Series - B: Physics and Biophysics, Veljko Zlatic (Editor), Alex Hewson (Editor). ISBN: 9789400749863 (2012), arXiv:1301.3307

[30] Tsung-Han Lee, Kenneth Yi-Jie Zhang, Chung-Hou Chung, Stefan Kirchner, Phys. Rev. B 88, 085431 (2013).

[31] D. L. Cox and A. L. Ruckenstein, Phys. Rev. Lett. 71, 1613 (1993).

[32] Y. Meir and Ned S. Wingreen, Phys. Rev. Lett. 68, 2512 (1992); Ned S. Wingreen and Y. Meir, Phys. Rev. B 49, 11040 (1994)

[33] M. Vojta, Phys. Rev. Lett. 87, 097202 (2001).

[34] Tsan-Pei Wu, Mater Thesis, Department of Electrophysics, National Chiao-Tung University, 2014.

[35] Imke Schneider, Lars Fritz, Frithjof B. Anders, Adel Benlagra, Matthias Vojta, Phys. Rev. B 84, 125139 (2011).

[36] C.H. Chung et al., in preparation. 